La collaboration scientifique franco-argentine est très vivante aujourd'hui, tant à l'échelle de grands projets bi- ou multinationaux qu'à celle d'échanges de quelques chercheurs.

Nous rappellerons d'abord la place privilégiée occupée de tout temps par les relations scientifiques franco-argentines, s'appuyant sur une forte influence culturelle française en Argentine. Nous présenterons ensuite, à titre d'exemple, une coopération développée depuis une trentaine d'années jusqu'à la formation d'un Laboratoire international associé. Enfin, nous examinerons les perspectives et les modalités ouvertes aujourd'hui pour les coopérations scientifiques avec ce pays.

Nous remercions Étienne Guyon et Charles de Novion pour leurs suggestions.

Les acronymes suivis d'un astérisque sont explicités dans le glossaire, p. 29.

\title{
La coopération scientifique franco-argentine Une longue histoire
}

José Eduardo Wesfreid ${ }^{(1)}$ (wesfreid@pmmh.espci.fr), Irene Ippolito ${ }^{(2)}$, Marta Rosen $^{(2)}$

et Jean-Pierre Hulin ${ }^{(3)}$

(1) Laboratoire PMMH, ESPCI, 10 rue Vauquelin, 75231 Paris Cedex 05.

(2) Grupo de Medios Porosos, Facultad de Ingeniería, Universidad de Buenos Aires, Paseo Colon 850, Buenos Aires (Argentine). (3) Laboratoire FAST, Bâtiment 502, Campus Paris-Sud, 91405 0rsay.

\section{Déjà, au $19^{e}$ siècle}

Il y eut tout d'abord, en Argentine, les grands voyageurs du dix-neuvième siècle, généralement envoyés en mission par l'Académie des sciences ou le Muséum, afin de récupérer des échantillons de la flore et de la faune. Les cas les plus connus sont ceux d'Aimé Bonpland (compagnon d'Alexandre de Humbold lors de l'expédition mémorable de 1799 à 1804, dans plusieurs autres régions d'Amérique, voir figure 1) et d'Alcide d'Orbigny, qui arrivent en mission scientifique avant Charles Darwin [1]. En 1817, le botaniste Aimé Bonpland (1773-1858) est nommé professeur d'histoire naturelle à Buenos Aires et, dès son arrivée, commence à explorer l'Amérique du Sud. Le naturaliste Alcide d'Orbigny, envoyé par le Muséum, part en 1826 passer sept ans dans cette région (voir figure p. 30). Durant son voyage, il va collecter, observer, décrire des échantillons dans tous les domaines de la zoologie des invertébrés comme des vertébrés, de la botanique, de l'anthropologie et de l'ethnologie. Les formidables collections qu'il rassemble, riches de presque 9000 espèces, très souvent nouvelles, sont expédiées directement au Muséum.

L'Argentine accède à l'indépendance en 1816 et se constitue en République, sous sa forme actuelle, en 1853. Elle crée ses

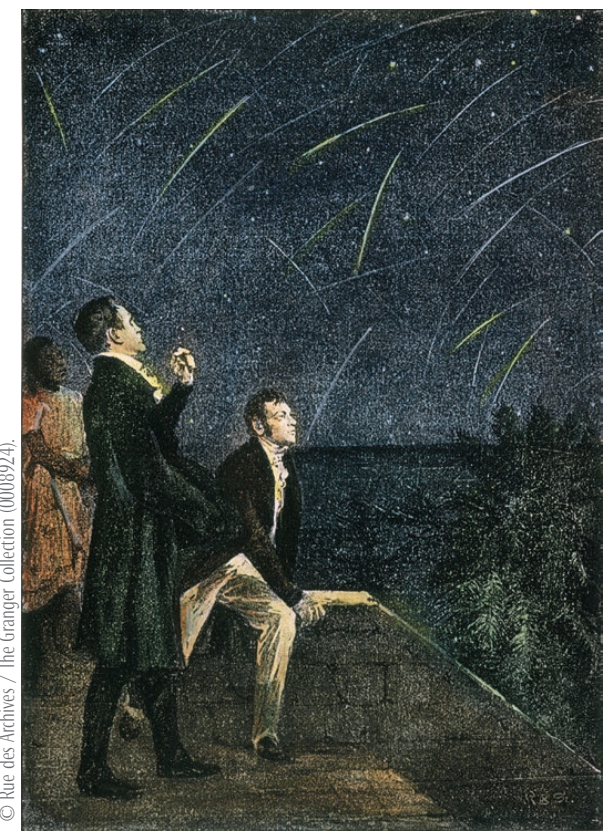

1. Alexandre von Humboldt et Aimé Bonpland observant une pluie de météores à Cumana, sur la côte nord de l'Amérique du Sud, le 12 novembre 1799.

premières institutions scientifiques en faisant appel à plusieurs universitaires et scientifiques français. C'est ainsi qu'en 1857 Auguste Bravard, géologue et paléontologue, est nommé directeur du Musée National de la Confédération Argentine à la ville de Parana, et Francis Beuf, lieutenant de l'armée française et directeur de l'observatoire de la Marine de Toulon, devient le premier directeur de l'Observatoire Astronomique de La Plata, fondé en 1881. En même temps, Amédée Jacques, normalien et philosophe, devient le Directeur du Colegio Nacional de Buenos Aires (fig. 2), le lycée le plus prestigieux d'Argentine [2]. 


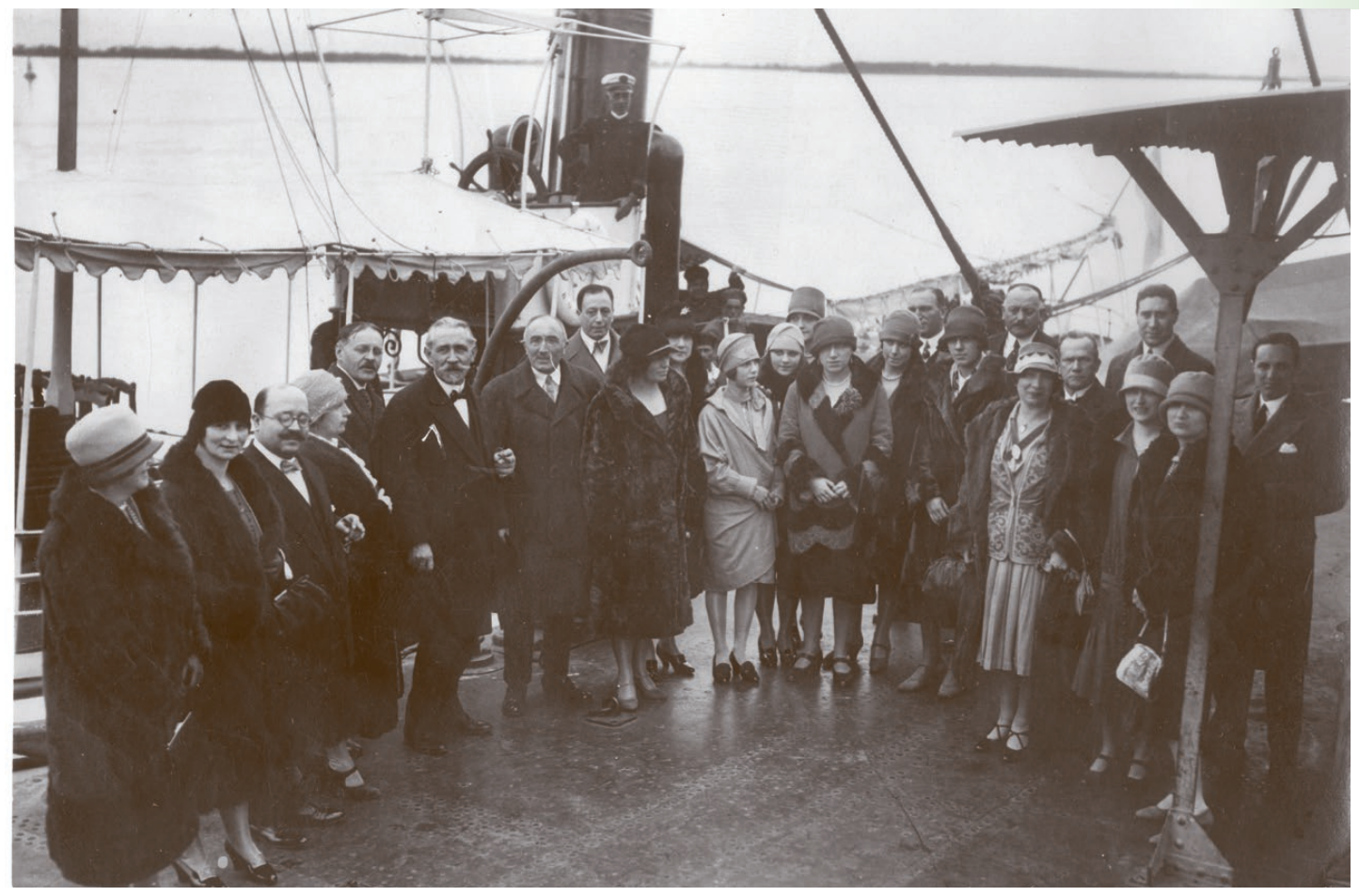

3. (a) Visite par Paul Langevin ( 6 e à partir de la gauche) du port de la ville de Rosario, en Argentine, avec des membres de la Faculté de Sciences de l'Université Nationale du Littoral. (Source : Archivo General de la Nación - Argentine, gentillesse de Maria Fuentes).

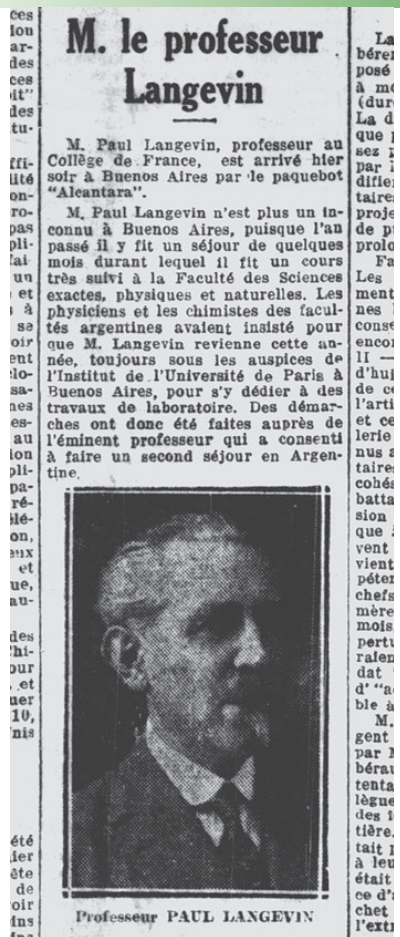

3. (b) Article du Courrier de la Plata du 17 juillet 1928, annonçant l'arrivée de Paul Langevin à Buenos Aires.

\section{Puis, au $20^{\mathrm{e}}$ siècle}

Plus récemment, Paul Langevin s'est fortement investi sous ces latitudes. Au cours de deux voyages, en 1927 et 1928 , il se rend à Buenos Aires pour donner, chaque fois, une série de neuf conférences, à l'invitation de l'Institut de l'Université de Paris à Buenos Aires [3] (fig. 3a). Cette institution, antenne informelle de la Sorbonne en Argentine, organisait régulièrement dans ce pays des missions d'un ou deux mois des plus éminents scientifiques français. L'Argentine, pays riche à l'époque, aimait également montrer son opulence en invitant des visiteurs illustres.

P. Langevin donna ainsi une série complète de cours à la Faculté des Sciences sur la relativité et la physique moderne, mais aussi à la Faculté de Philosophie. Le moment était particulièrement bien choisi car, déjà en 1925, Albert Einstein s'était rendu en Argentine, et avait donné des conférences sur le même sujet, bien que la communauté des physiciens de l'époque fût très réduite (c'est seulement en 1944 qu'est fondée l'Association Physique de l'Argentine). La répercussion des cours de Langevin fut considérable et donna lieu, le lendemain de chaque conférence, à une chronique régulière dans le quotidien La Prensa. La répercussion sociale ne fut pas moindre et Le Courrier de La Plata, quotidien français

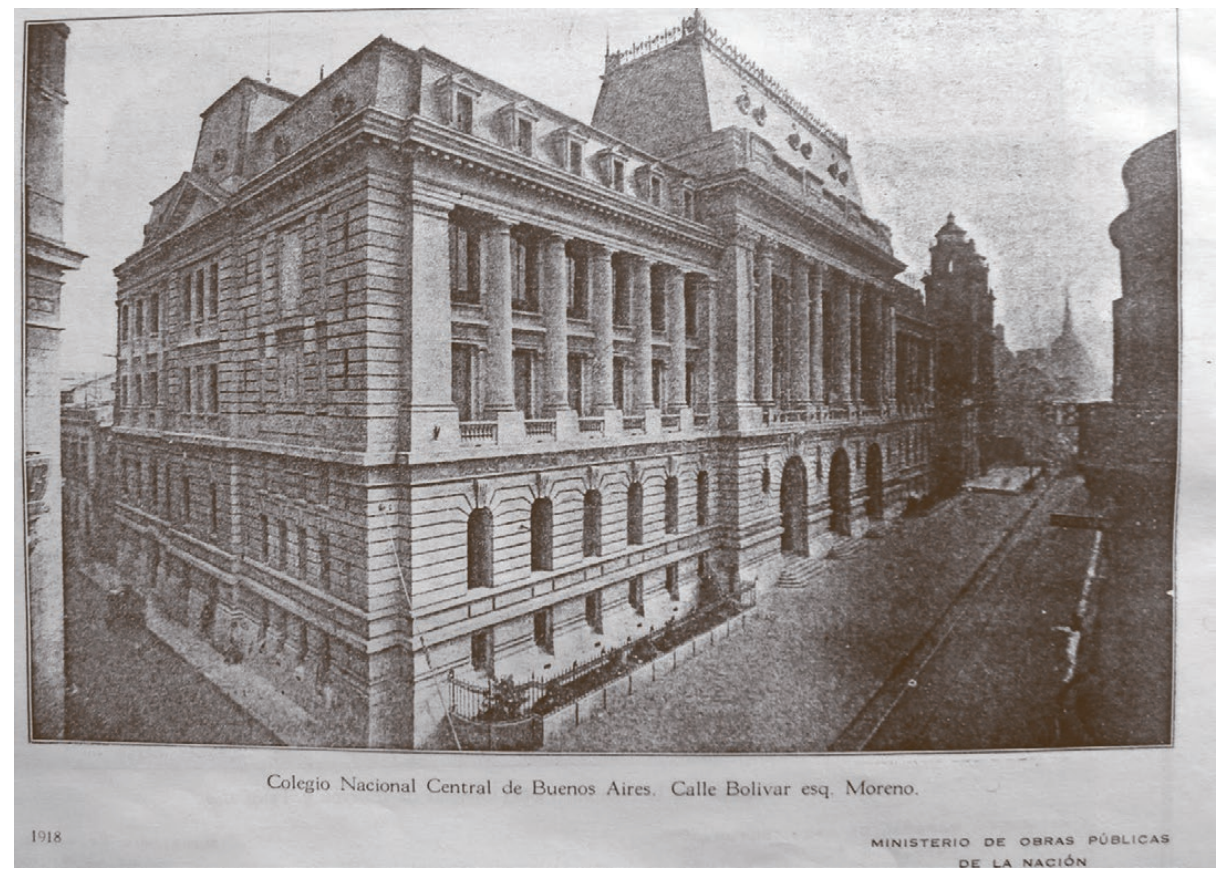

2. Le Colegio Nacional Central de Buenos Aires, en 1918.

de Buenos Aires, couvrit régulièrement les déplacements de Langevin (fig. 3b) qui allaient d'une visite à l'Hôpital Français jusqu'à un dîner en ville.

Les relations du scientifique français avec ses homologues argentins allaient plus loin que des missions. Le professeur Horacio Damianovich, de la ville de Santa Fé, fut visiteur au Collège de France, et c'est avec lui que Langevin organisa à Paris, la réunion internationale de Chimie Physique en
1928. Ce congrès, sous la présidence de Jean Perrin, fut l'un des premiers à réunir, à Paris, des chercheurs allemands et alliés, remettant ainsi en cause le boycott décidé par la Conférence des Académies des Sciences interalliées, au lendemain de la Première Guerre mondiale [4]. Langevin se félicite que "cette initiative née en Argentine, [soit] une contribution au rapprochement de scientifiques [et rappelle] le rôle de la science comme facteur de paix ».

>>> 


\section{Des échanges dans les deux sens}

La route de la science n'a pas du tout été à sens unique entre la France et l'Argentine : ainsi, Florentino Ameghino, paléontologue et anthropologue argentin, qui effectua de très nombreuses découvertes de grands animaux fossiles en Argentine, séjourne en France entre 1878 et 1881 , où il rédige avec Henri Gervais, du Muséum, sa première monographie sur ces mammiferes [5]. Il explora également les traces de l'homme quaternaire dans les carrières de Chelles, en 1880 [6].

En 1931, deux Argentins, Fernando Pérez, créateur d'une méthode d'analyse des peintures par lumière rasante et ambassadeur d'Argentine en Italie, et Carlos Mainini, professeur à l'Université de Buenos Aires et attaché scientifique à Paris, furent à l'origine de la création du Laboratoire de Recherches du Louvre (aujourd'hui Centre de Recherche et de Restauration des Musées de France - C2RMF) et en devinrent les animateurs [7] (fig. 4).

Plusieurs fois dans l'histoire, la mobilité scientifique entre la France et l'Argentine a également été motivée par des raisons politiques. C'est ainsi que A. Bravard et A. Jacques, partisans de la Révolution de février 1848, s'exilent en Argentine après le coup d'état bonapartiste de décembre 1851. Dans l'autre sens, la France a accueilli un groupe de scientifiques argentins à la suite

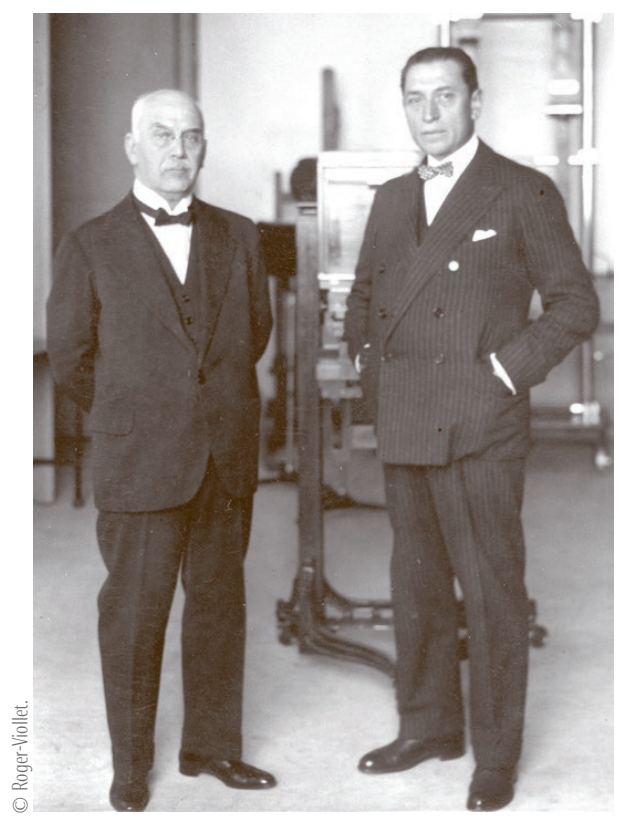

4. Fernando Perez (à gauche) et Carlos Mainini à l'inauguration, en 1931, du Laboratoire du Musée du Louvre-Institut Mainini. du coup d'État de 1966 et, plus particulièrement, de la "Nuit des Longs Bâtons ", où l'armée a investi et occupé l'Université Argentine. La répression s'est alors abattue sur les enseignants de la Faculté des Sciences, motivant la démission de $80 \%$ des enseignants et l'exil de 301 d'entre eux. Plusieurs de ces chercheurs ont joué, en France, un rôle important dans la création de l'école moderne de biophysique et biologie moléculaire. Le dernier coup d'État, le plus sanglant de l'histoire argentine, a provoqué en 1976 l'exil de nombreux chercheurs argentins vers la France. L'un d'eux, Eduardo Pasquini, physicien à Saclay, rentra en Argentine au cours de la dictature et est, depuis, porté disparu, comme 31 autres physiciens, suite à son arrestation par les forces de répression [8]. À Paris, un "Comité pour la libération des physiciens argentins emprisonnés » est constitué, présidé par Alfred Kastler, prix Nobel.

\section{Un exemple de coopération}

C'est pendant la période de reconstruction de la démocratie, qui suivit la chute de cette dictature en 1983, que démarra en 1985 une coopération entre la Faculté d'Ingénierie de l'Université de Buenos Aires (UBA) et plusieurs laboratoires français. Cette action porte sur la physique de l'hydrodynamique et des milieux désordonnés, et donne une idée des différents modes de coopération possibles. À cette époque, le Groupe de milieux poreux (GMP) de cette faculté venait juste d'être créé et recherchait des coopérations extérieures : les difficultés pratiques pour monter une activité expérimentale étaient innombrables mais, par contre, des jeunes chercheur(se)s enthousiastes en stage de fin d'études universitaires représentaient un apport inestimable.

Des accords ponctuels de deux ans entre le CNRS et son équivalent argentin, le CONICET $^{\star}$, ainsi que des participations argentines à quelques écoles d'été (Cargèse, Les Houches) facilitèrent le démarrage de la coopération entre un laboratoire de l'École Supérieure de Physique et Chimie Industrielles (PMMH-ESPCI*) et le GMP sur des problèmes d'instabilités hydrodynamiques et de dispersion de traceurs dans les milieux poreux. Des missions de seniors dans les deux sens et des séries de conférences (É. Guyon) permirent des contacts avec des étudiant(e)s et aboutirent aux soutenances, en 1993 et 1995, de deux thèses d'étudiantes argentines, entièrement préparées au laboratoire PMMH : ces deux étudiantes (dont une des auteurs du présent article (I.I.)) sont maintenant enseignanteschercheuses à la faculté d'ingénierie et jouent un rôle clé dans la coopération.

L'étape suivante fut, à partir de 1992, la mise en place de plusieurs programmes PICS* financés par le CNRS : ces structures plus ambitieuses permettent de financer des séjours d'assez longue durée de jeunes chercheurs en cours de thèse [9]. Ce support a été, au départ, complété par le ministère des relations extérieures : ensuite, le programme ECOS-SUD* finança sur projets un certain nombre de missions. De plus, des postes d'un mois de professeurs invités, tant à l'ESPCI qu'aux Universités Pierre et Marie Curie et Paris Sud, facilitèrent des visites de seniors. Enfin, pendant quelques années, un programme CEE ciblé sur les pays en voie de développement compléta, entre autres, l'équipement et l'installation du laboratoire argentin et aida les laboratoires français : on pouvait encore, à cette époque, monter des projets européens à taille humaine sur des sujets ouverts !!!

L'ensemble de ces programmes a associé entre 1992 et 2007 le GMP et plusieurs laboratoires français (PMMH, FAST ${ }^{\star}$, $\left.\mathrm{GMCM}^{\star}, \mathrm{PMC}^{\star}\right)$. Cette coopération a permis de mener à bien six thèses en cotutelle (fig. 5) et trois thèses en coopération simple : six de ces docteurs sont maintenant enseignants-chercheurs au GMP. Pendant les thèses en cotutelle, particulièrement efficaces mais coûteuses en termes de missions, les étudiants effectuent des séjours de longue durée dans le pays d'accueil (un an à un an et demi au total) et reçoivent un double diplôme de thèse français et argentin. Cette procédure est soumise à des conditions strictes et requiert un accord préalable des deux universités. Elle n'a pas pu être suivie dans tous les cas et, dans certains, on a eu simplement une codirection formelle ou informelle des thèses à l'occasion de missions ou à distance, et des séjours de plus courte durée des doctorant(e)s. Ce volet "formation " s'est révélé crucial pour le développement de notre coopération et l'établissement de contacts étroits ; il s'est aussi traduit des deux côtés par un meilleur "plurilinguisme ", facilitant les échanges. Les thèses en coopération ont également permis le développement de nouvelles thématiques, ainsi que des ouvertures vers des applications industrielles. 


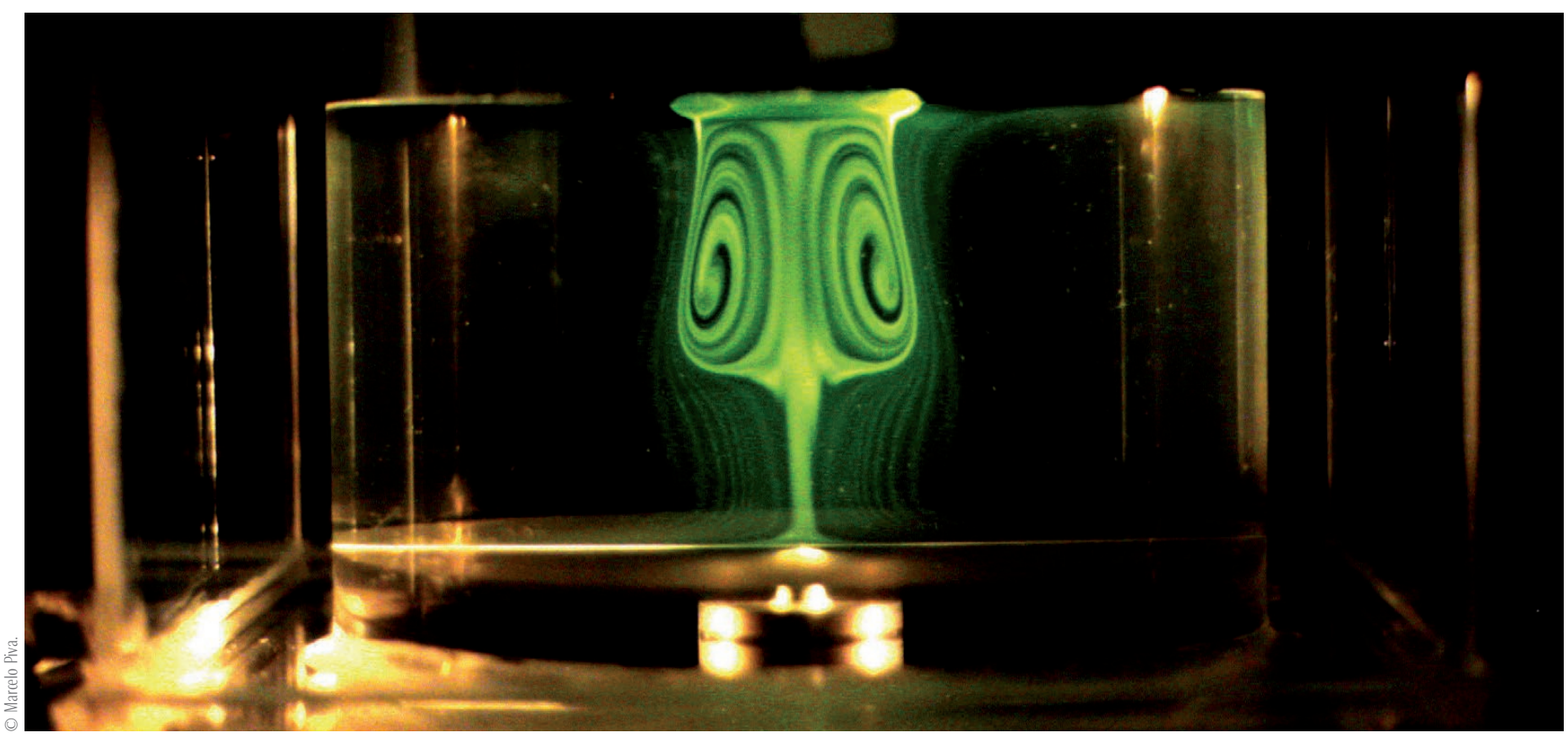

5. Apparition d'une cellule de recirculation dans un volume d'eau à surface libre contenu dans un récipient cylindrique, dont le fond tourne autour d'un axe vertical. L'écoulement, visualisé de côté par injection dans l'axe d'un colorant fluorescent, est observé dans un plan passant par l'axe de rotation. Cette étude du phénomène de séparation qui amène au “vortex breakdown", a été réalisée par Marcelo Piva, aujourd'hui Maître de conférences à l'UBA, dans le cadre d'une thèse en cotutelle dirigée par Adriana Calvo (Argentine) et José Eduardo Wesfreid (France).

Le bilan très positif, scientifique et humain, de cette coopération a permis de la renforcer et de lui donner une nouvelle dimension. Depuis 2010, une nouvelle étape a été franchie avec la création du Laboratoire International Associé (LIA $\left.{ }^{\star}\right)$ Physique et Mécanique des Fluides [10] : celui-ci inclut la coopération précédente et associe sur des sujets voisins, outre les laboratoires GMP, PMMH et FAST, les laboratoires d'Hydraulique et de Dynamique des fluides de la FIUBA $\star$, LIMSI^ d'Orsay et l'Institut PPrime` de Poitiers. Dans son dernier renouvellement, elle intègre également des équipes de la Faculté de Sciences de l'UBA et de l'ENS Lyon. Cette nouvelle structure apporte une meilleure visibilité, ainsi qu'un soutien mieux équilibré et plus permanent, tant du CNRS que du CONICET argentin. Outre le financement du CNRS, des bourses ont pu être obtenues pour des doctorants ou postdoctorants (bourses Bernardo Houssay, du nom d'un prix Nobel de médecine argentin) ; plusieurs enseignants-chercheurs argentins ont également bénéficié de postes de professeurs invités de nos organismes universitaires de rattachement. Des financements supplémentaires, par exemple à travers des appels d'offres conjoints entre les deux pays par l'ANR et l'ANPCyT ${ }^{\star}$, seraient cependant indispensables.

\section{Glossaire}

ANPCyT : Agencia Nacional de Promoción Científica y Tecnológica, équivalent argentin de I'Agence Nationale de la Recherche française (ANR).

CONICET : Consejo Nacional de Investigaciones Científicas y Técnicas, organisme argentin équivalent au CNRS.

ECOS : organisme assurant pour les ministères des Affaires étrangères et ceux de l'Enseignement supérieur la coordination de la coopération scientifique avec des pays d'Amérique latine ; ECOS-Sud concerne le cône sud de l'Amérique (Argentine, Chili, Uruguay). Depuis sa création, près de 300 projets ont été financés avec l'Argentine.

FAST : Laboratoire Fluides, Automatique et Systèmes Thermiques, Campus d'Orsay.

FIUBA : Faculté d'Ingénierie de I'Université de Buenos-Aires.

GMCM : Groupe Matière Condensée et Matériaux, maintenant intégré à l'Institut de Physique de Rennes, Campus Beaulieu, Rennes.

Institut PPrime : Recherche et ingénierie pour les transports et l'environnement (Poitiers).

LIA : Laboratoire International Associé ; cette structure inclut des membres de plusieurs laboratoires français et étrangers pour une durée de 4 ans, renouvelable sur un projet scientifique.

LIMSI : Laboratoire d'Informatique pour la Mécanique et les Sciences de I'Ingénieur, Campus d'Orsay.

PICS : Programme International de Coopération Scientifique cofinancé, par exemple, par le CNRS et le CONICET, et finançant pendant trois ans des échanges de scientifiques entre au moins deux laboratoires.

PMC : Laboratoire de Physique de la Matière Condensée (Collège de France).

PMMH-ESPCI : Laboratoire de Physique et Mécanique des Milieux Hétérogènes (École Supérieure de Physique et Chimie Industrielles de la Ville de Paris).

UMI : Unité Mixte Internationale, implantée sur un seul site et cogérée par plusieurs organismes de recherche français et étrangers (CNRS et CONICET, entre autres). Une UMI peut avoir son propre personnel appartenant aux deux nations. 


\section{Hier et aujourd'hui}

Pendant les années 50-60 et le début des années 1970 puis, de nouveau, après la chute de la dictature, de nombreuses autres coopérations ont été établies entre la France et l'Argentine.

Ainsi, pendant la première période, dans le domaine du nucléaire, le CEA et son équivalent argentin, la CNEA, ont établi de fortes coopérations où le CEA a accueilli pour des séjours longs plusieurs chercheurs argentins dans le domaine des matériaux nucléaires, y compris sur des aspects fondamentaux.

De plus, en 1964, l'Argentine désira acheter un premier réacteur nucléaire et entreprit une négociation pour la cession d'un réacteur français de la filière UNGG (uranium naturel graphite gaz), négociation qui a été brusquement interrompue [11], probablement à cause du refus du ministère français des Finances.

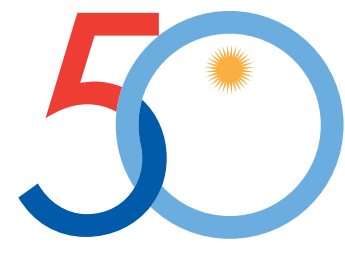

FRANCIA - ARGENTINA $5 O$ AÑOS DE COOPERACIÓN

6. Logo édité en 2014 , pour le $50^{\mathrm{e}}$ anniversaire du traité de coopération scientifique franco-argentin.
Le cadre institutionnel moderne de la coopération franco-argentine a été lancé il y a tout juste 50 ans (fig. 6), à l'occasion d'une longue visite du Général de Gaulle en Amérique Latine. A ainsi été signé, le 3 octobre 1964 à Buenos Aires, le traité de coopération scientifique, technique et culturelle, élargi à partir de 1997 par la signature d'un accord complémentaire.

Aujourd'hui, la coopération scientifique franco-argentine est forte de plus d'une centaine d'accords. Ainsi, la France est devenue le premier partenaire de l'Argentine en matière de coopération scientifique bilatérale et le troisième pays d'accueil des étudiants argentins [12].

Ces coopérations vont d'accords limités à quelques voyages d'échange d'un ou deux chercheurs sur deux ans jusqu'à une unité mixte internationale (UMI CNRSCONICET) sur le climat. Outre le LIA mentionné plus haut, cinq autres ont été créés dans les domaines de l'informatique, des nanotechnologies et des neurosciences [13]. Des accords particuliers sur de très grands instruments (synchrotron Soleil, observatoire Auger...) et les micro-nanotechnologies ont également été signés, pour ne parler que de la physique. Il existe le programme ARFITEC de coopération scientifique entre les grandes écoles et les facultés d'ingénierie, et d'autres programmes de coopération sont actuellement en projet, comme le CAFCI (centre binational en sciences de l'ingénieur).
Il ne faudrait cependant pas perdre de vue que c'est à travers la continuité des relations de chercheur(se) à chercheur(se), et des échanges de longue durée de jeunes en formation, que peuvent se construire des coopérations réellement fructueuses, tant sur le plan scientifique qu'humain. I

\section{Références}

1 Les naturalistes français en Amérique du Sud, XVI'-XIXe siècles, sous la direction d'Y. Laissus, Éditions du CTHS (2005).

2• P. Vermeren, Le rêve démocratique de la philosophie D'une rive à l'autre de l'Atlantique, L'Harmattan (2001).

3• D. Quattrochi-Woisson, Un nationalisme de déracinés. L'Argentine, pays malade de sa mémoire, Éditions du CNRS (1992).

4- B. Schroeder-Cudehus, Les scientifiques et la paix, Presses de l'université de Montréal (1978).

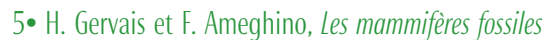
de l'Amérique du Sud, Librairie F. Savy, Paris (1880).

6• F. Ameghino, Bulletins de la Société d'anthropologie de Paris, III ${ }^{\circ}$ Série, tome 3 (1880), pp. 638-646.

7• http://www.c2rmf.fr/

8• "Hommage à Eduardo Pasquini », Reflets de la Physique n²0 (juillet-août 2010), p. 30

9• Voir site du CNRS : www.dgdr.cnrs.fr/daj/ international/structures.htm

10• http://liapmf.limsi.fr/index-fr.html

11• J.A. Sabato, World Development, 1, n̊8 (1973) 23-38.

12- www.embafrancia-argentina.org/La-cooperationscientifique-et

13 - Listes de LlAs et de coopérations en cours avec l'Argentine : http://www.cnrs-ameriquelatine.d/ paises/argentine.asp

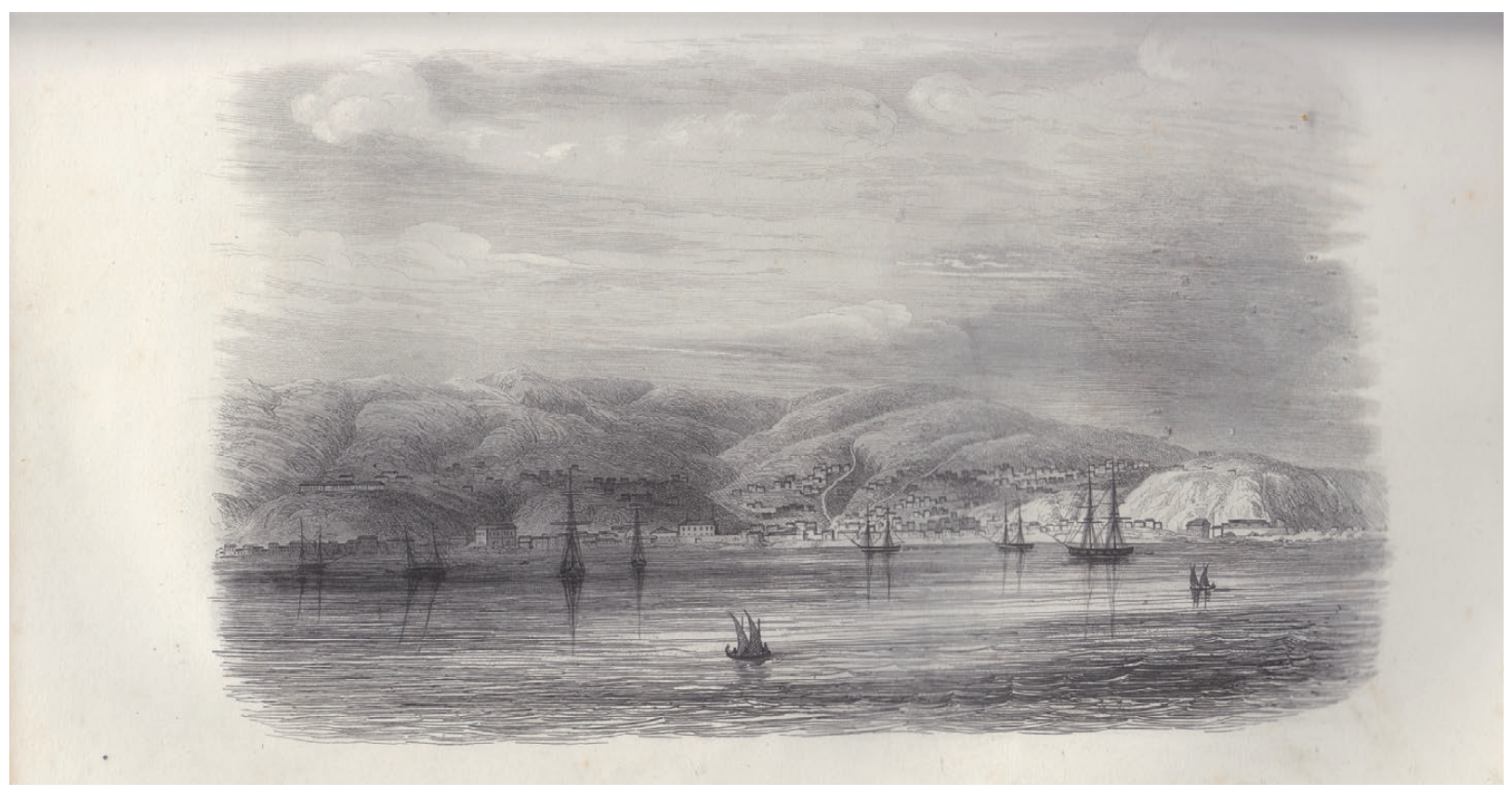

